In Situ

Revue des patrimoines

\section{In Situ}

Revue des patrimoines

$14 \mid 2010$

Le cavalier du Pont-Neuf : histoire, restauration et secrets de la statue équestre de Henri IV

\title{
Restauration de la statue de Henri IV
}

\section{Stéphanie Celle et Carlo Usai}

\section{OpenEdition}

\section{Journals}

Édition électronique

URL : http://journals.openedition.org/insitu/6989

DOI : $10.4000 /$ insitu.6989

ISSN : 1630-7305

Éditeur

Ministère de la culture

Référence électronique

Stéphanie Celle et Carlo Usai, « Restauration de la statue de Henri IV », In Situ [En ligne], 14 | 2010, mis en ligne le 10 avril 2012, consulté le 19 avril 2019. URL : http://journals.openedition.org/insitu/6989 ; DOI : 10.4000/insitu.6989

Ce document a été généré automatiquement le 19 avril 2019

\section{(c)}

In Situ Revues des patrimoines est mis à disposition selon les termes de la licence Creative Commons Attribution - Pas d'Utilisation Commerciale - Pas de Modification 4.0 International. 


\title{
Restauration de la statue de Henri IV
}

\author{
Stéphanie Celle et Carlo Usai
}

\section{Introduction}

1 Le Service Départemental de l'Architecture et du Patrimoine de Paris (SDAP) assure les travaux d'entretien d'un certain nombre de monuments classés appartenant à l'État dont trois statues familières aux Parisiens : la statue de Jeanne d'Arc située Place de Pyramide, la statue de Louis XIV placée au centre de la Place des Victoires et la statue de Henri IV dominant le square du Vert Galant sur le Pont Neuf.

2 Dans la continuité des opérations précédentes sur les statues de Jeanne d'Arc et de Louis XIV, fut réalisée à l'automne 2004 la restauration de la statue en bronze de Henri IV et de son socle en marbre. Ces travaux ont été effectués sous la maîtrise d'ouvrage de la Direction régionale des affaires culturelles d'île-de-France et en collaboration avec le Laboratoire de recherche des monuments historiques.

3 Cette intervention était importante parce que les recherches archivistiques préparatoires ont permis de penser qu'il s'agissait de la première opération d'ensemble depuis la création de l'œuvre. On allait pouvoir observer de près et d'une façon exhaustive la statue pour recueillir des informations sur son mode d'élaboration et sur son histoire.

4 Ce type de travaux peut être confié à un fondeur et le mode d'intervention consistera alors en une réfection à neuf de la patine à chaud comme il fut fait lors de la création de statue et en son ultime étape. Mais il fut proposé de s'inscrire dans le droit fil de la philosophie de Cesare Brandi qui a défini la patine comme partie intégrante de l'œuvre et comme élément témoignant de l'histoire singulière d'un objet et plus précisément, de sa surface.

5 L'objectif de la restauration était donc de respecter la patine naturelle du bronze ; surface certes altérée au cours du temps mais authentique. Dans le but de préserver cette authenticité de l'œuvre, il était indispensable de procéder à une analyse fine de l'état de la surface afin de dégager l'ensemble des enjeux de l'intervention et de définir un 
protocole adéquat permettant de répondre aux différents problèmes tout en conservant la patine naturelle.

6 Ce chantier offrait également une occasion rare pour explorer l'intérieur de la statue. Cette effigie du Bon Roi fut restituée sous la Restauration. Divers écrits parlaient de documents déposés dans l'antre en bronze lors de la cérémonie officielle d'inauguration du monument.

\section{Travaux préparatoires}

Les travaux préparatoires à la restauration ont permis d'énoncer les enjeux multiples de l'intervention; enjeux explicités dans le cahier des charges adressé aux entreprises qualifiées lors de l'appel d'offre. Ces enjeux découlent d'une compréhension de l'adéquation entre le matériau dont est constituée la statue et le milieu environnant dans lequel elle est située.

Figure 1

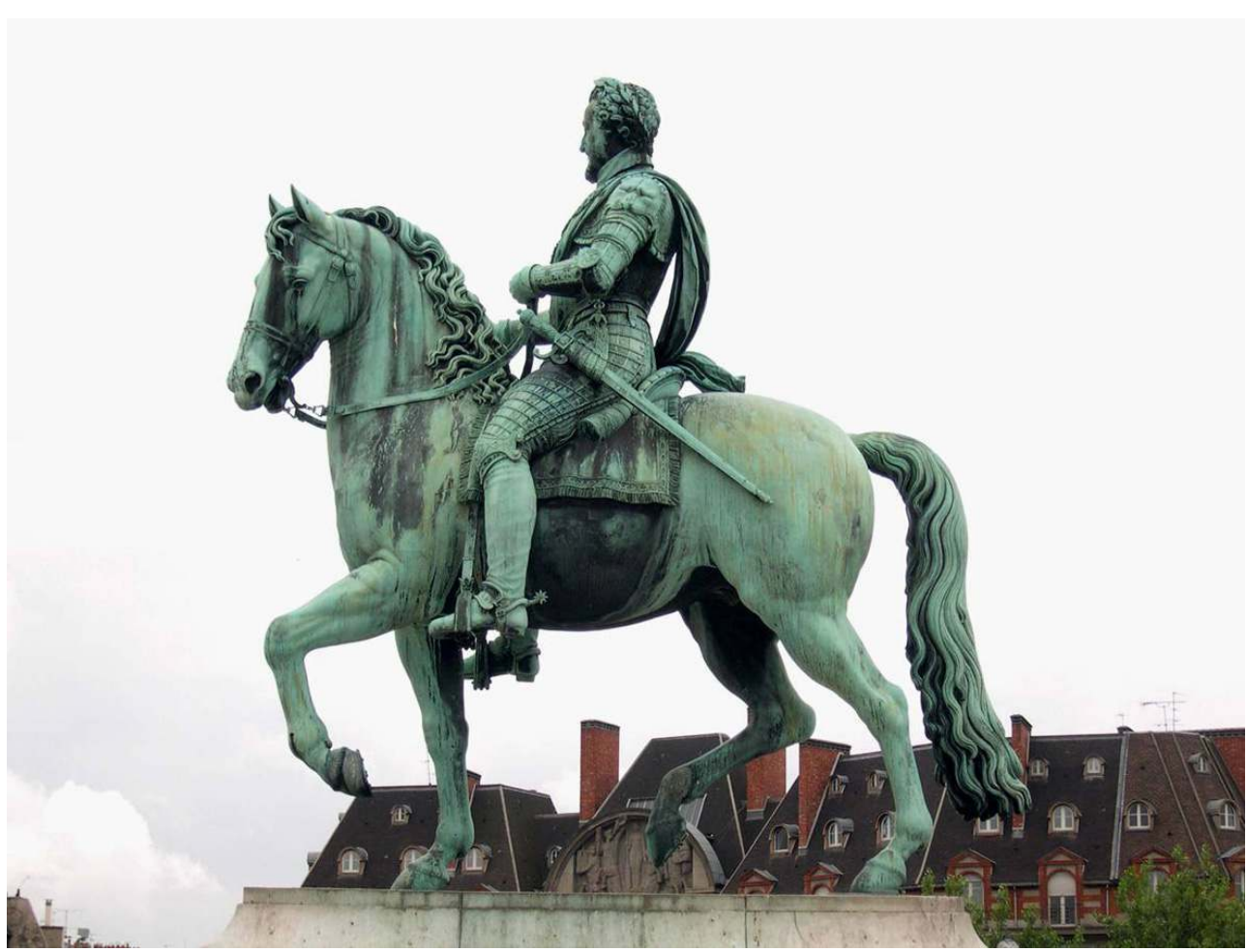

État des surfaces de la statue avant la restauration

Phot. C. Usaï, 2004

La statue est en bronze, il s'agit précisément d'un alliage formé de quatre métaux dans les proportions suivantes : cuivre $84 \%$, étain 3,5\%, plomb 5,5\% et zinc 4,9\%. Le bronze est un corps instable qui va forcément chercher à se modifier en surface pour adopter un état minéral plus stable, et le caractère de cette mutation est fonction de l'environnement et de ses composantes physico-chimiques. La transformation de la surface du bronze est donc une évolution inéluctable dont la nature et l'aspect dépendent du milieu; elle aboutit à la formation de la patine naturelle du bronze, plus ou moins stable selon les cas (fig. $n^{\circ}$ 1). 
Figure 2

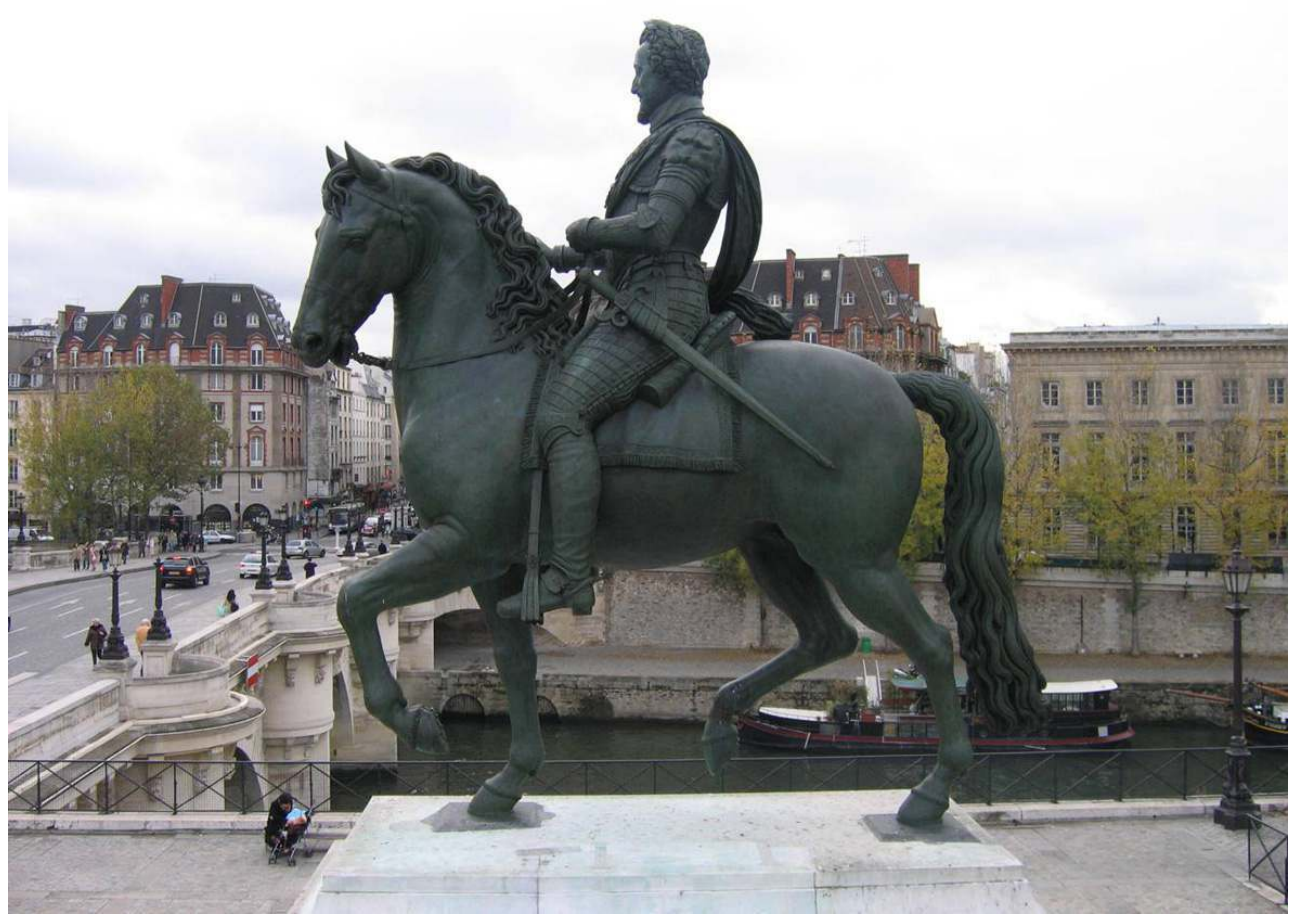

État des surfaces de la statue après la restauration

Phot. C. Usaï, 2004

Dans un contexte exempt de pollution, les bronzes adoptent une patine dont la forme chimique tend vers le cristal de malachite, couche compacte qui protège le métal. Cette couche confère une tonalité vert-sombre. Et cette tonalité reste la référence culturelle pour les œuvres réalisées dans ce noble alliage (fig. $\mathbf{n}^{\circ} \mathbf{2}$ ). Cet aspect de surface ne peut pas être le résultat du travail naturel du temps sur la matière car l'atmosphère est en fait chargée de particules de pollution qui ont pour conséquence la fabrication d'une patine d'une autre nature, portant atteinte à la conservation du métal. C'est ce phénomène que nous pouvions observer sur la statue de Henri IV avant sa restauration : la patine due à deux siècles de transformation patente de la surface du bronze dans un environnement urbain pollué.

\section{Analyse de la surface}


Figure 3

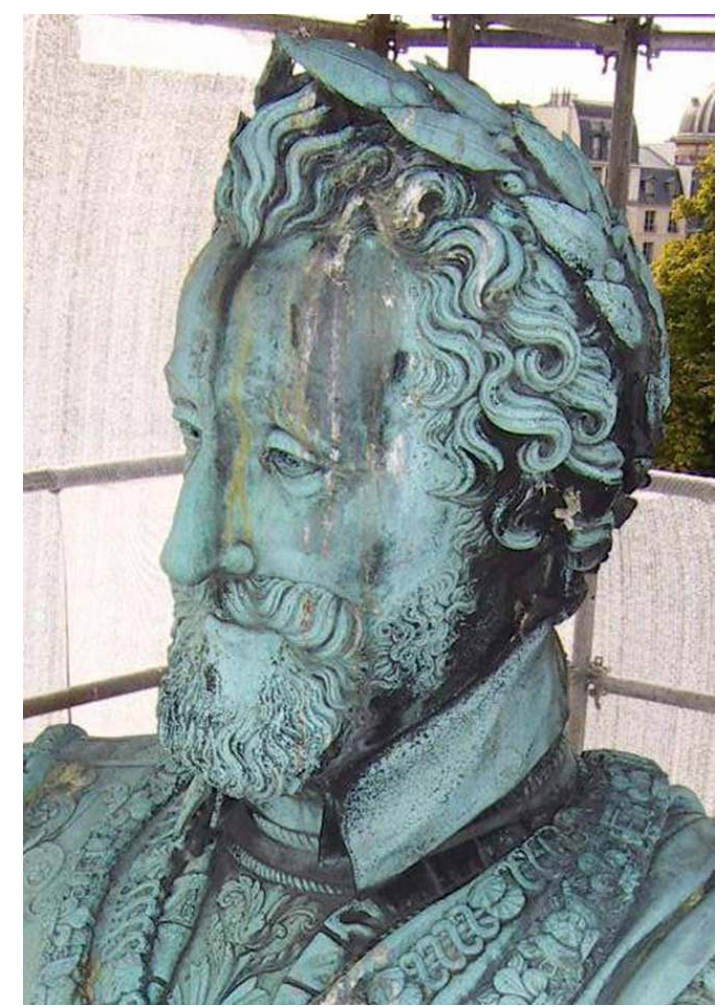

Détail de la tête avant traitement

Phot. C. Usaï, 2004

Une observation à l'œil nu de l'ensemble de la statue permet d'opposer dans un premier temps deux types de surfaces: les surfaces vert-clair et les surfaces noir-sombre. Les surfaces vert-clair sont situées sur les parties de la statue exposées aux eaux de pluie et sur les zones de cheminement des eaux de ruissellement : le flanc, la croupe du cheval, les parties supérieures et latérales des membres du cavalier.... (fig. $\mathbf{n}^{\circ}$ 3) La transformation de ces surfaces est donc liée à l'action de l'eau de pluie dont on connaît le caractère légèrement acide en milieu urbain. Au contraire, les surfaces noir-sombre correspondent aux parties de la statue protégées des eaux : le ventre du cheval, les parties en creux du cavalier, les plis du relief... Dans ce cas, l'encrassement des surfaces n'est pas perturbé par le lessivage des eaux de ruissellement; des dépôts de poussières et des particules de charbon s'accumulent progressivement jusqu'à constituer une couche noire indurée (fig. $\mathbf{n}^{\circ}$ 4). 


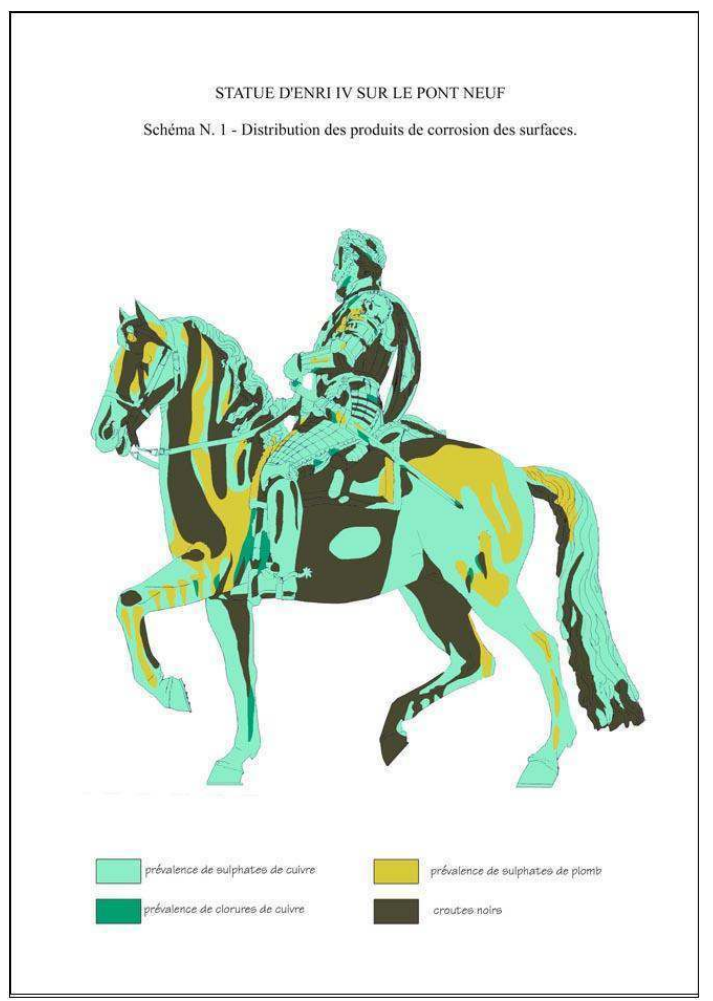

Schéma sur l'état des surfaces avant traitement

Phot. C. Usaï, 2004

11 L'observation au microscope d'une coupe en surface permet de comprendre les phénomènes de constitution des différentes patines précédemment identifiées. Un échantillon prélevé à partir d'une surface vert-clair fait apparaître une couche brunrouge au contact du métal: la cuprite résultant d'une première oxydation en surface. Cette couche de cuprite est recouverte d'une couche vert-clair dont le niveau extérieur est en creux par rapport au niveau originel de la surface du bronze jusqu'à former parfois des micro-cratères. Cette couche vert-clair correspond au phénomène de corrosion du métal sous l'action de l'acidité des eaux de ruissellement. La corrosion est réductrice de matière puisque le bronze est « rongé » en surface et diminue en épaisseur au profit de la formation d'un produit de corrosion essentiellement constitué de sulfate de cuivre. Selon les situations, cette couche de corrosion d'un aspect alors décliné, peut également contenir en grand nombre des sulfates de plomb (aspect ocre jaune), des chlorures de cuivre (aspect bleu-vert) et d'autres sels, produits dus à la dégradation des métaux composant l'alliage (fig. $\mathbf{n}^{\circ}$ 5). Globalement, cette altération de nature peu compacte constitue en surface de la statue une couche poreuse et hydrophile qui, en retenant l'eau, est un facteur d'accélération du processus de dégradation. Du point de vue de la conservation du matériau, plus cette couche vert-clair est épaisse, plus elle est néfaste. 
Figure 5

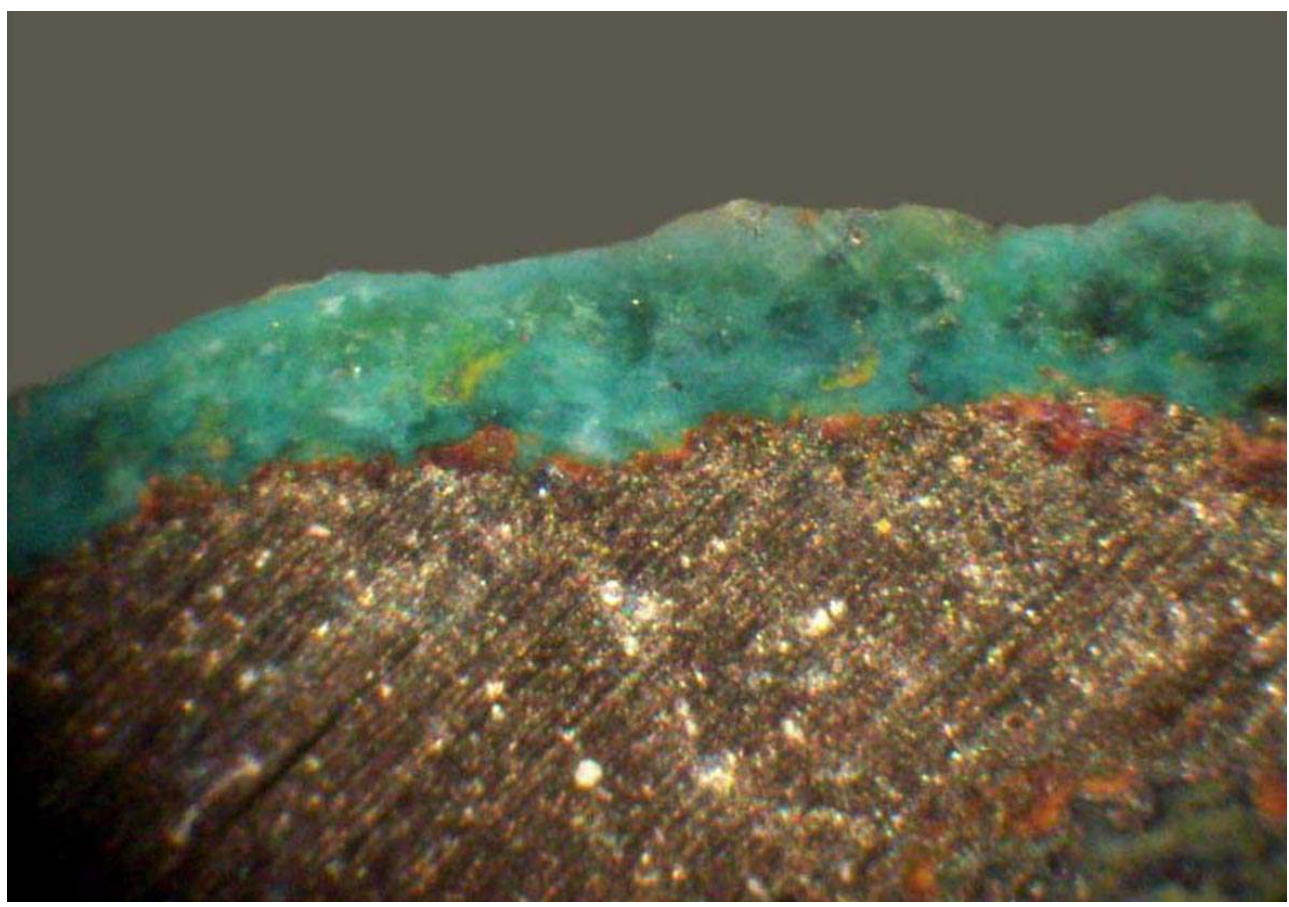

Coupe transversale sur le bronze, produit de corrosion en surface

Phot. C. Usaï, 2004

12 Une coupe au microscope réalisée à partir d'une surface noire fait apparaître au-dessus de la couche de cuprite une couche indurée se superposant à la surface originelle de la statue et figurant un phénomène de sédimentation. Cette croûte noire est constituée d'éléments assimilables à du charbon ainsi que de différents sels solubles dont les concentrations sont moindres que celles rencontrées dans la couche vert-clair. D'une certaine façon, la croûte noire peut aussi être considérée comme protectrice de la surface originelle puisqu'elle la recouvre en reproduisant toutes les traces inscrites sur la matière-matrice telles que celles laissées par le passage des râpes utilisées en phase de finition.

D'autres analyses, réalisées par diffraction X à partir de prélèvements en différents points de la patine, ont permis de distinguer quatre principaux types de produits d'altération : les sulfates de cuivre (antlérite, brocantite) de couleur bleu-vert, les sulfates de plomb (anglésite) de couleur ocre, les chlorures de cuivre (atacamite) eux aussi de couleur bleu vert, et un dernier mélange d'atacamite, d'antlérite, de gypse et de particules carboniques, de couleur noire. 


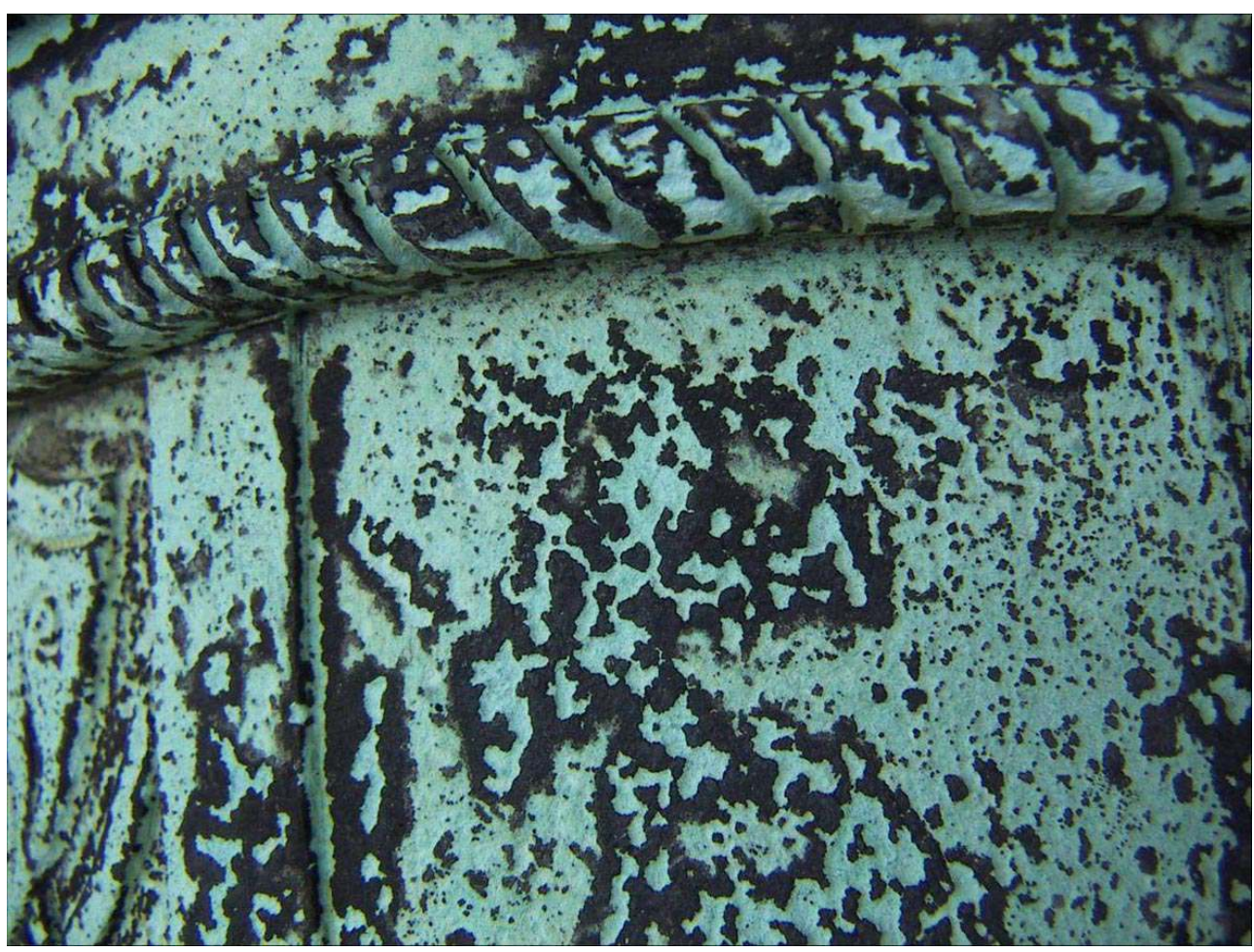

Formation de cratères dus à la corrosion

Phot. C. Usaï, 2004

14 On peut dire également que la répartition des surfaces vert-clair et des surfaces noirsombre dépend de leur situation et plus exactement du modelé de la statue. Des nuances pourraient être apportées car le relief définit des zones plus ou moins exposées aux eaux de pluie, ce qui entraîne une action inégale de l'eau et des variations multiples dans l'état des surfaces. En certains endroits très altérés, on trouve combinées les surfaces vert-clair et les surfaces noires; et ces dernières semblent alors comme de petits îlots en relief piquetant la surface corrodée (fig. $\mathbf{n}^{\circ} \mathbf{6}$ ).

\section{Enjeux de la restauration}

On peut dire que l'état de la surface dont nous héritons est lié à la situation d'une matière particulière, le bronze, dans un environnement spécifique, urbain, et que la transformation qui s'opère dans le temps est aussi fonction du relief de la statue et de son exposition aux intempéries. En fait, rien n'est dû au hasard dans la transformation de cette surface, tout est fonction des conditions physico-chimiques comme si elles étaient mises en équation. Le même constat peut être fait à tous les niveaux de lecture de la statue. 
Figure 7

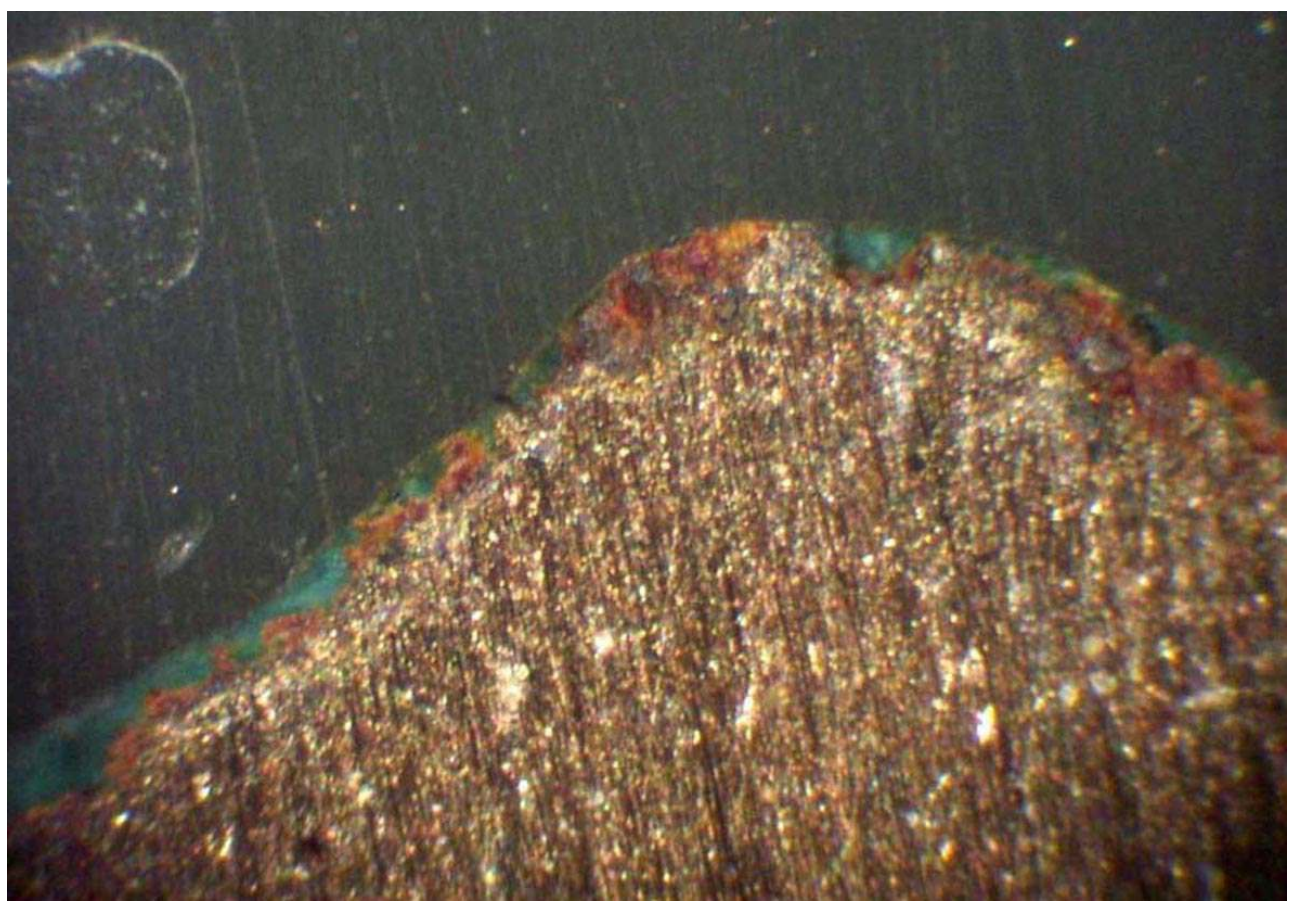

Coupe transversale sur le bronze, diminution du produit de corrosion après traitement

Phot. C. Usaï, 2004

16 L'intervention doit répondre à un premier enjeu qui est celui de la conservation de la statue ; c'est-à-dire chercher à ralentir au mieux l'inéluctable travail de la corrosion qui entraîne une réduction de l'épaisseur du métal. Pour ralentir la corrosion, il faut rendre la surface du bronze moins réceptive à l'eau en augmentant son imperméabilité (fig. $\mathbf{n}^{\circ} 7$ )

L'intervention doit également répondre à des exigences esthétiques et permettre une mise en valeur de l'œuvre dont la perception est altérée par le manque d'unité de la surface. Avant la restauration, on observe de forts contrastes entre les zones claires et les zones sombres et ces contrastes gênent la vision de l'œuvre dans son ensemble. Le cheval semble fragmenté entre ses antérieurs et postérieurs vert-clair et son ventre noir. En plusieurs endroits comme sur le tapis de selle, on distingue des bandes verticales noires qui barrent les surfaces et qui correspondent à des zones non lessivées où un encrassement s'est effectué. Sur le mollet par contre, l'effet de blanchiment de cette partie très corrodée tend à effacer certains modelés. La précision du travail de ciselure est perdue à cause des multiples produits de corrosion qui brouillent la vision du relief dans son détail. Afin de redonner à voir l'œuvre d'art dans son ensemble mais aussi afin de retrouver la qualité originelle de la surface sculptée, les contrastes entre les zones claires et les zones foncées doivent être réduits au mieux à l'échelle du tout et à l'échelle du détail.

Le troisième enjeu de l'intervention est un enjeu patrimonial lié à la notion d'héritage, de transmission d'un objet ancien auquel le temps aurait donné une valeur ajoutée: la patine. Ces deux siècles écoulés ont façonné la sculpture, lui ont conféré une patine naturelle qui fait à présent partie de l'histoire de la matière constitutive de l'œuvre. La patine naturelle de la surface est le résultat de la combinaison des conditions intrinsèques 
de la matière et de son relief et des conditions subies du milieu extérieur. Effacer la patine, c'est effacer deux siècles d'histoire, ne pas en garder le témoignage. Afin de respecter le passage du temps et la patine naturelle, l'intervention est réalisée en conservation de la surface et de la répartition de ses nuances.

Tels que définis précédemment, l'enjeu conservatoire, l'enjeu esthétique et l'enjeu patrimonial paraissent être des objectifs paradoxaux puisque les produits de corrosion sont jugés de façon négative ou positive selon les points de vue. Mais c'est l'importance de l'épaisseur de la couche vert-clair qui la rend hydrophile, c'est la force trop grande des contrastes qui perturbe la lecture, ce sont donc certains des caractères de ces produits qui devront être modifiés sans remettre en cause leur présence puisqu'ils constituent également la patine. Le protocole d'intervention cherche le meilleur compromis pour concilier l'ensemble des objectifs; c'est un travail sur la nuance et le double aspect des choses.

\section{Protocole d'intervention}

Figure 8

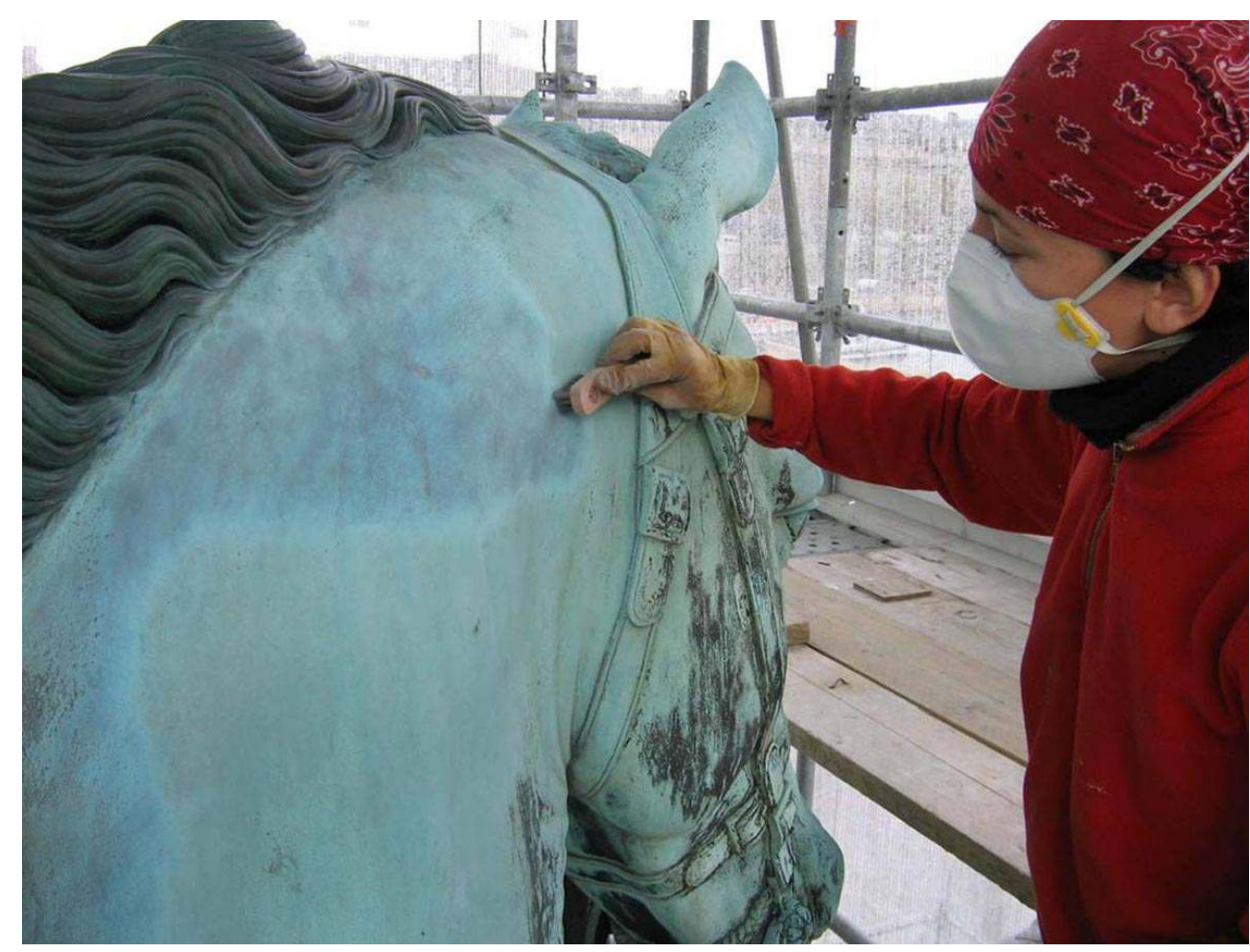

Nettoyage sélectif de la surface par brossage

Phot. C. Usaï, 2004 
Figure 9

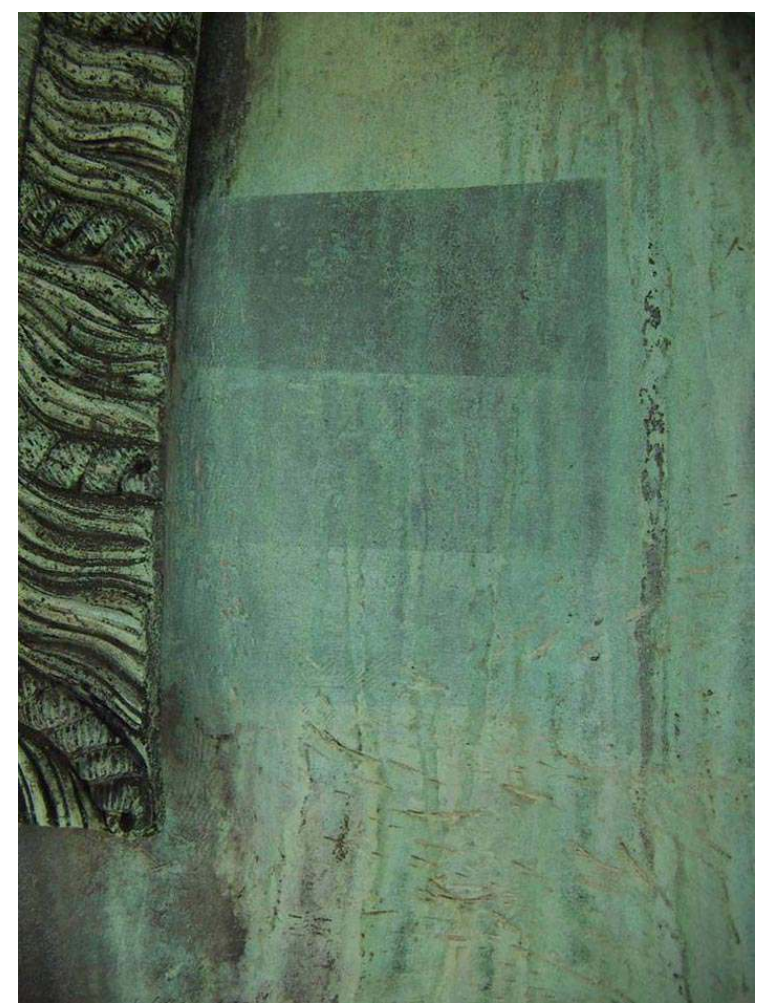

Test montrant différents niveaux de brossage

Phot. C. Usaï, 2004

20 La première phase du protocole d'intervention, est une phase de nettoyage mécanique manuel des surfaces à l'aide de brosses métalliques souples de tailles diverses. Le geste manuel peut être modulé et différents niveaux de nettoyage obtenus (fig. $\mathbf{n}^{\circ} \mathbf{8}, \mathbf{n}^{\circ} \mathbf{9}$ ). Pour les surfaces vert-clair, cette phase cherche à éliminer la surépaisseur pulvérulente et poreuse tout en préservant une mince couche de patine en superficie. Le brossage des zones noires permet d'affiner la croûte et d'en adoucir les contours (fig. $\mathbf{n}^{\circ} \mathbf{1 0}$ ). 
Figure 10

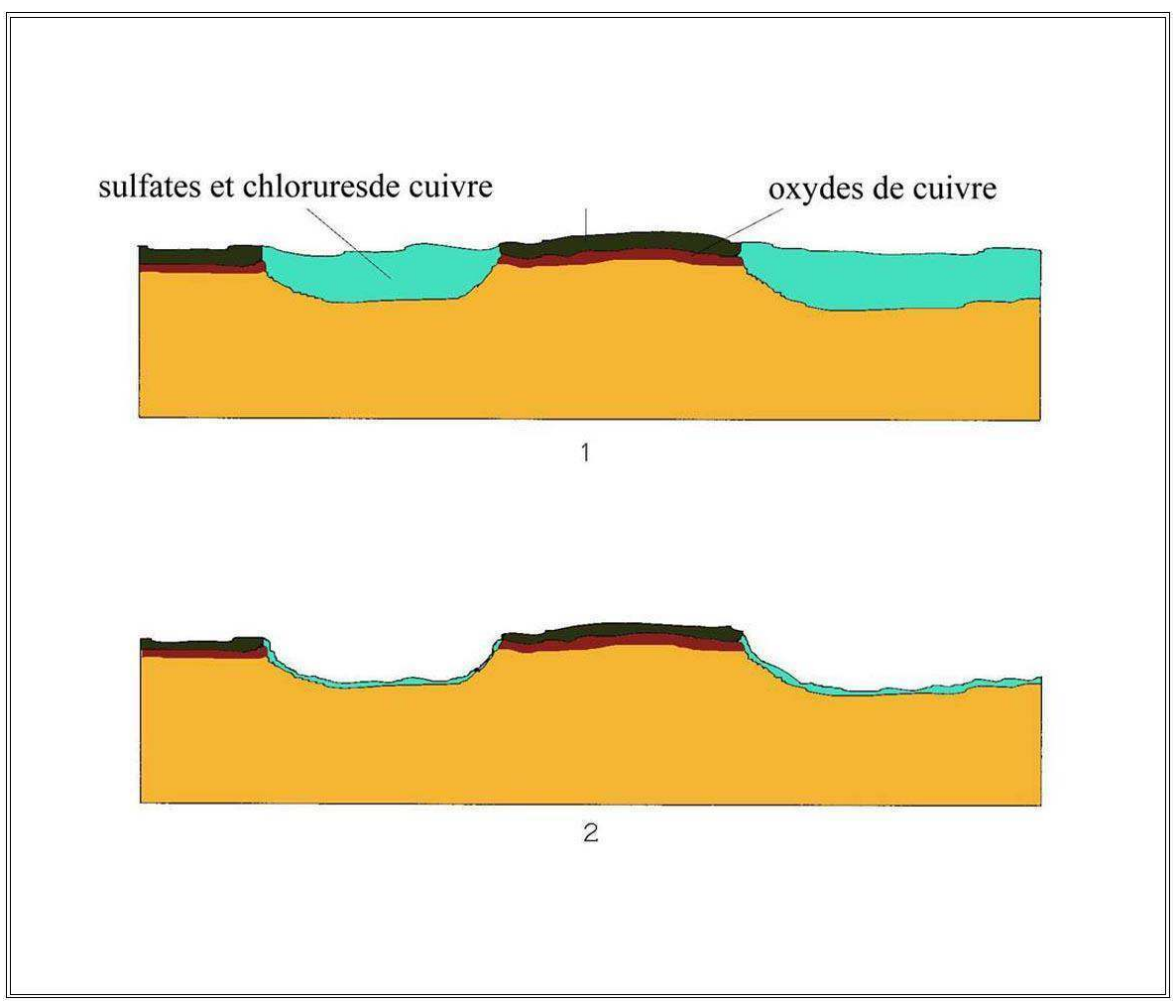

Coupe schématique expliquant le protocole d'intervention

Phot. C. Usaï, 2004

21 Après le nettoyage mécanique, l'ensemble des surfaces est abondamment rincé à l'eau distillée. Cette phase favorise l'élimination des sels solubles et leur taux de concentration dans l'eau sale doit tendre vers une valeur basse et régulière pour donner les garanties de l'efficacité du rinçage. 
Figure 11

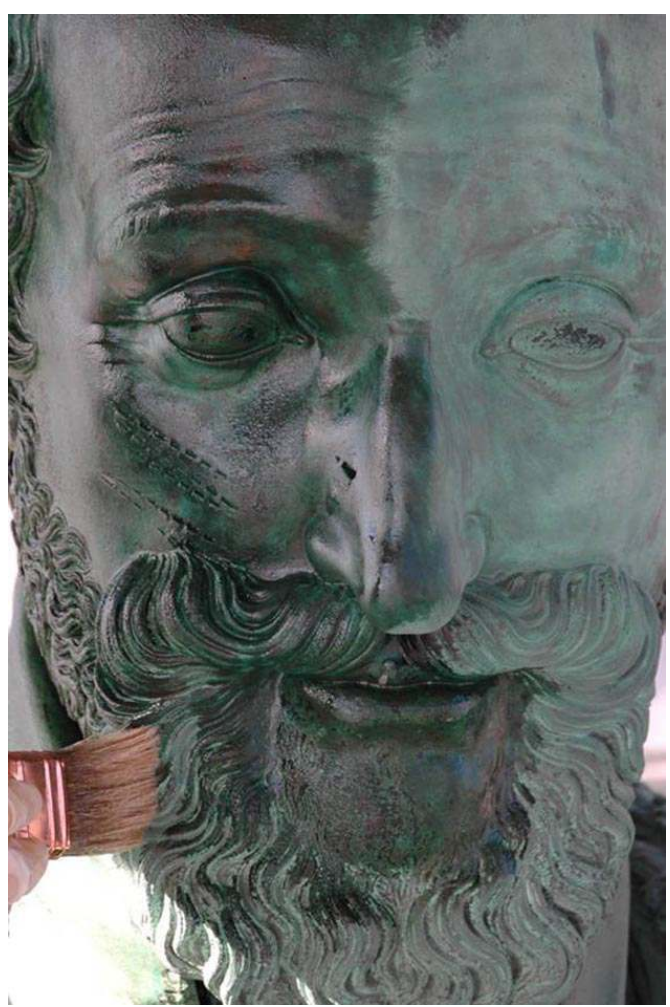

Détail de la tête, application d'une couche protective Phot. C. Usaï, 2004

Le protocole se poursuit par une phase visant à ralentir le travail de corrosion à l'aide d'un inhibiteur de corrosion : le benzotriazole utilisé dans une solution d'alcool éthylique (2\%) (fig. $\left.\mathbf{n}^{\circ} \mathbf{1 1}\right)$. Une application généreuse de cette solution est réalisée au pinceau pour mettre les surfaces en condition de «bain ». Le benzotriazole est absorbé par les produits de corrosion et réagit avec ceux-ci afin de former des sels stables qui permettront de constituer une couche protectrice. Mais cette action a pour effet de modifier la couleur des surfaces en leur conférant un aspect assombri et luisant, comme "mouillé». La concentration de la solution en benzotriazole, le ph du milieu et le temps d'immersion de la surface traitée sont les facteurs de l'efficience inhibitrice du traitement et les facteurs de modification de l'aspect de la surface. Ils doivent être mesurés en fonction des situations différenciées afin d'obtenir une protection et une harmonisation des surfaces.

À la phase d'inhibition fait suite une phase de protection du métal par la superposition de deux films appliqués l'un après l'autre au pinceau sur toute la superficie. L'objectif est d'éviter les contacts entre le bronze et l'eau (des précipitations atmosphériques et de l'humidité ambiante) en intercalant entre le métal et le milieu extérieur, une couche imperméable.

24 Le premier film est constitué par l'application d'un vernis acrylique adjuvanté de benzotriazole, produit commercialisé sous le nom d'Incralac (CTS). Ce produit est réversible et se dissout commodément avec de l'acétone. Sous la dilation du métal, ce premier film pourrait se micro-fissurer, ce qui confèrerait à la surface un effet de craquelures. 
Figure 12

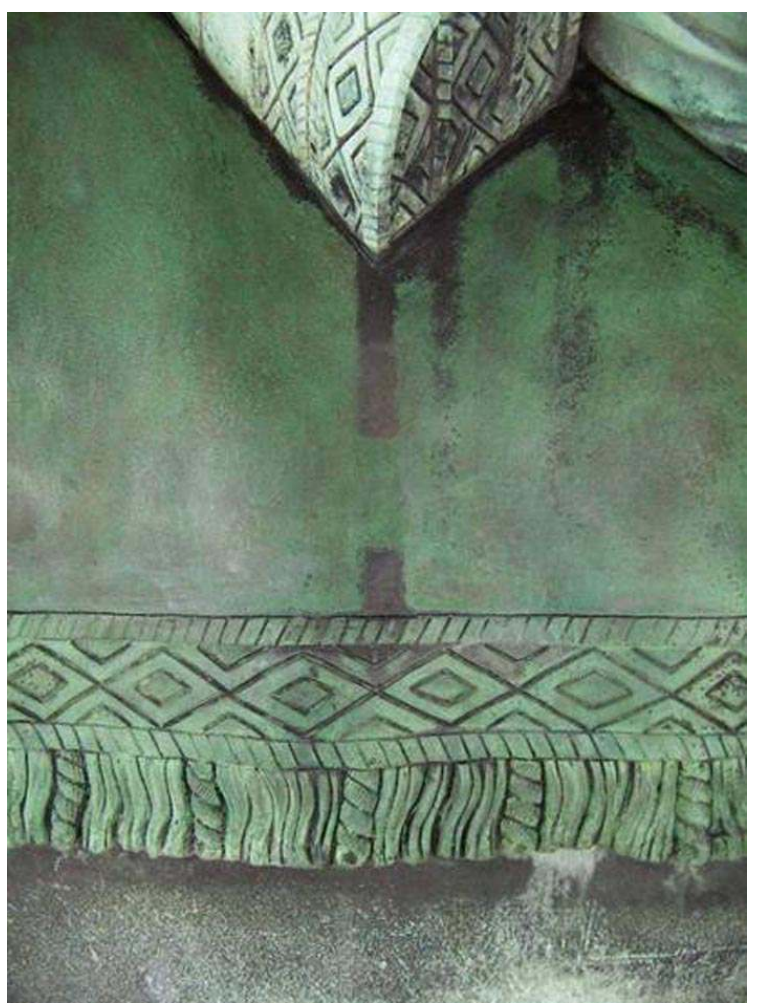

Coulure noire masquée par l'ajout de pigments dans la couche protective

Phot. C. Usaï, 2004

Le second film protecteur doit répondre à deux problèmes : l'un est conservatoire et l'autre est esthétique. Ce film doit protéger l'Incralac pour éviter son usure et son craquellement. Il doit également permettre des corrections chromatiques très ponctuelles en masquant certaines tâches noires demeurées trop prégnantes et jugée gênantes pour la lecture de l'œuvre (fig. $\mathbf{n}^{\circ} \mathbf{1 2}$ ). Ce second film protecteur de l'Incralac et correcteur de couleur si nécessaire, est réalisé avec une cire micro-cristalline (par exemple la cire micro-cristalline Reswax-Spica) additionnée ou non à des pigments naturels et à des terres colorées selon les tonalités souhaitées. Il s'agit d'une couche sacrificielle qui devra être renouvelée régulièrement sur les zones lessivées par l'eau de pluie. Cet entretien peut être annuel ou d'une périodicité plus ou moins espacée suivant la sollicitation des surfaces (fig. $\left.\mathbf{n}^{\circ} \mathbf{1 3}\right)$. 


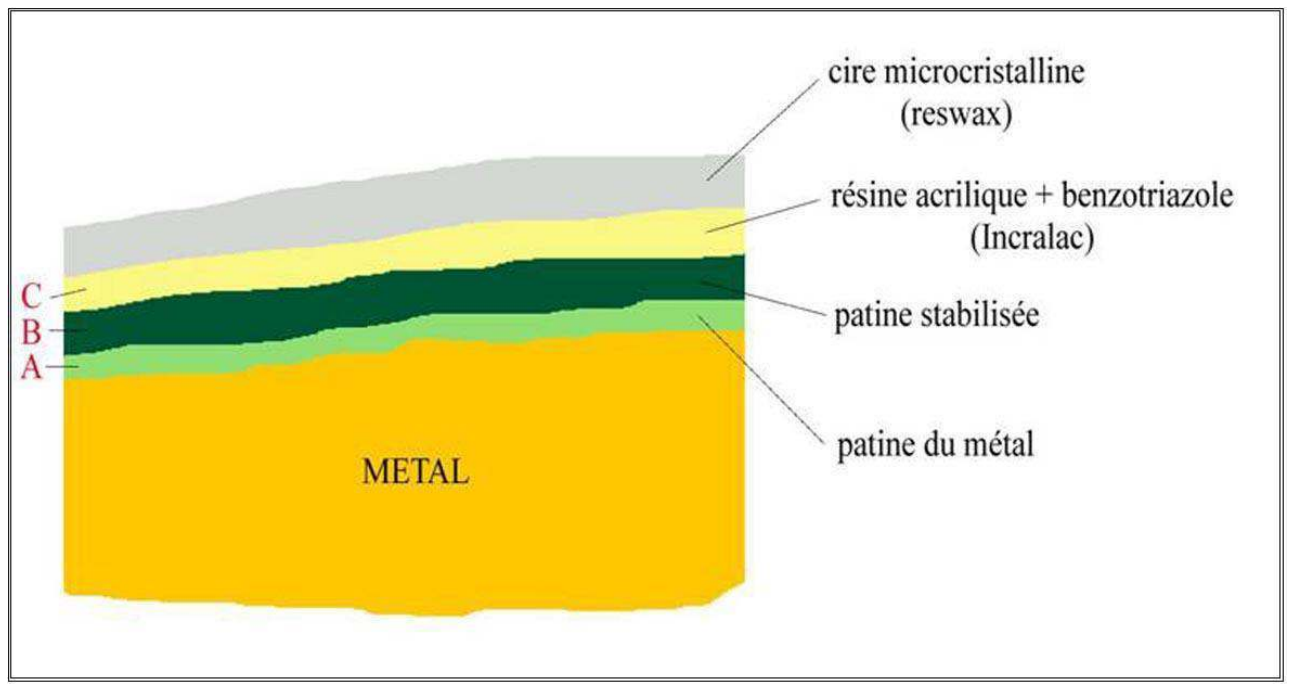

Schéma explicatif du protocole de conservation

Phot. C. Usaï, 2004

Par ailleurs, des petites cavités ont été identifiées en surface du bronze. D'un diamètre allant jusqu'à $2 \mathrm{~cm}$, elles résultent de bulles d'air formées lors de la fonte. Les plus profondes, qui constituent des réceptacles d'eau et de poussières présentent un risque pour la conservation, ont été rebouchées avec un mélange de résine époxy et de terres naturelles. D'autres cavités sont le fruit d'une usure du matériau et ont été colmatées afin d'éviter leur agrandissement.

\section{Restitution de l'éperon droit}




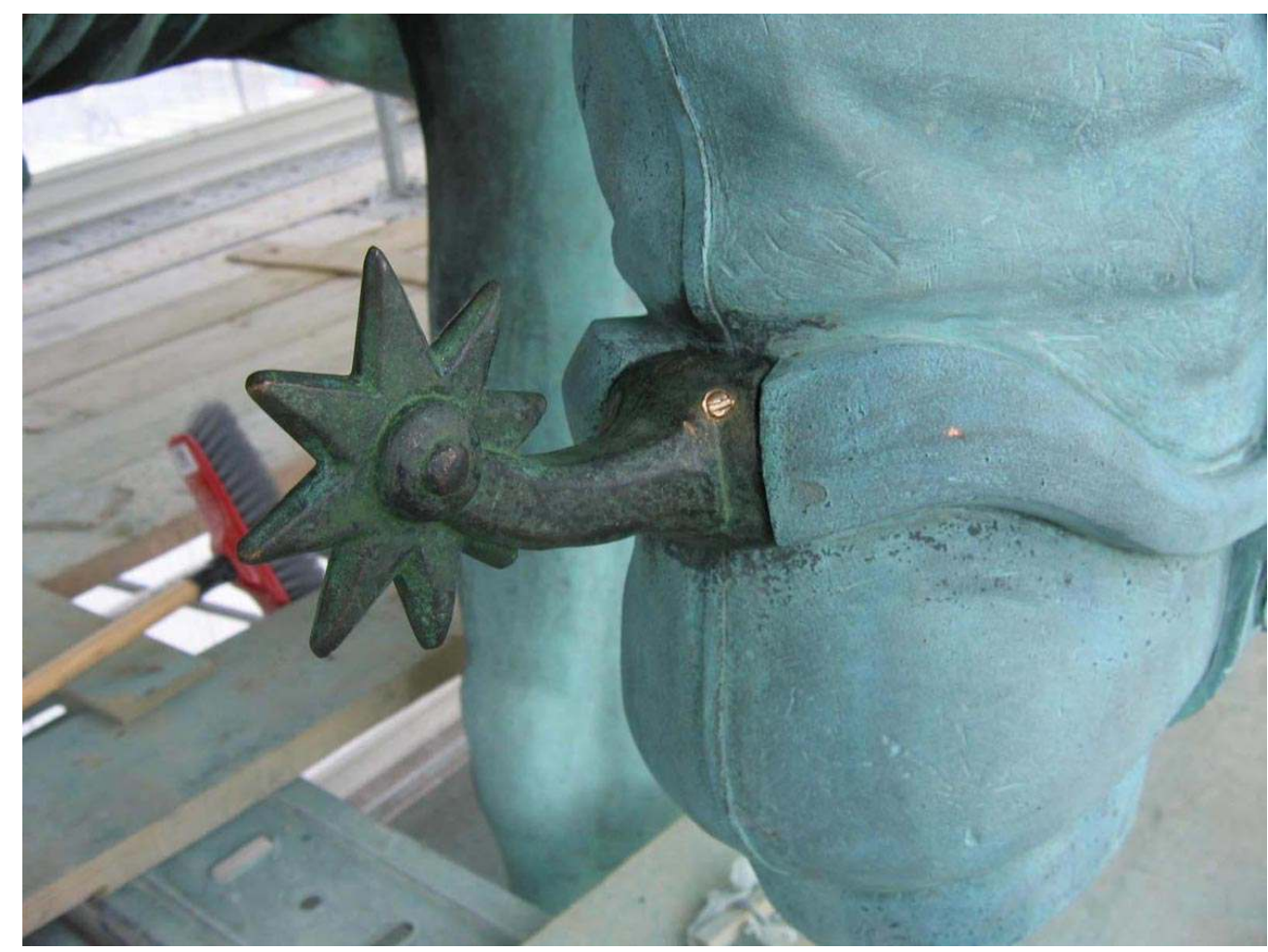

Détail de l'éperon restitué

Phot. C. Usaï, 2004

Le cavalier avait perdu son éperon au talon droit depuis fort longtemps. Ce petit détail avait déjà retenu l'attention d'Alexandre Dumas, observateur auquel rien n'échappait, qui s'en servit dans une scène d'ivresse nocturne sur le Pont-Neuf de son roman «Vingt ans après », ouvrage publié en 1845 . Pour restituer cet élément, une empreinte de l'éperon gauche a été réalisée et a permis de faire un modèle en cire. Ce modèle servit d'âme pour la fonte en bronze du nouvel éperon droit par la méthode de la cire perdue (fig. $\mathbf{n}^{\circ} \mathbf{1 4}$ ).

\section{Cas des bas-reliefs}

Le bronze des bas-reliefs est un alliage différent de celui de la statue; il est constitué de : $85 \%$ de cuivre, $0,6 \%$ d'étain, $11,2 \%$ de plomb et $1,9 \%$ de zinc. Il s'agit d'un bronze pratiquement sans étain et riche en plomb; ce dosage en fait un matériau plus tendre et plus facile à travailler à froid pour le ciseleur qui assure les interventions de finition. Avant restauration, les surfaces des bas-reliefs présentaient d'une façon générale, une patine plus sombre que celle de la statue avec un fond noir qui effaçait le travail fin de sculpture. La situation en léger renfoncement des ouvrages inclus dans le socle, explique la formation d'un encrassement important et l'étendue de cette croûte noire.

Le brossage manuel a permis de retrouver la nervosité du dessin en débarrassant les surfaces des produits pulvérulents et en désépaississant la couche de patine. L'application d'une cire pigmentée sur l'arrière-plan des bas-reliefs a permis une harmonisation des tonalités et une réintégration de ces parties au sein des scènes représentées. 
Lors de cette intervention, la baguette défaillante encadrant le bas-relief gauche a été complétée en partie haute; cette baguette en bronze est un couvre-joint qui assure l'étanchéité de la jonction entre le support et le bas-relief en applique.

\section{Observation de la surface de la statue}

Un chantier de restauration c'est aussi un moment privilégié pour la connaissance de l'œuvre et de son histoire propre. L'échafaudage permet d'approcher la surface et d'en faire une observation exhaustive; le nettoyage de cette surface permet d'y faire réapparaître des informations, des traces, des détails qu'il faudra interpréter.

La signature du sculpteur se trouve sous le sabot de l'antérieur gauche levé où l'on peut lire : F.F. Lemot, 1818. Les deux bas-reliefs sont signés de la même façon en partie basse avec cependant la datation de 1820. La signature du ciseleur Mesnel se distingue incisée sur le harnais du cheval de la statue. Pour les bas-reliefs, on retrouve la signature de l'artisan sur les créneaux des fortifications de Paris et sur le haut de la tête du cheval de Henri IV. Les initiales « E.J. » sont également présentes sur le bas-relief Sud.

Figure 15

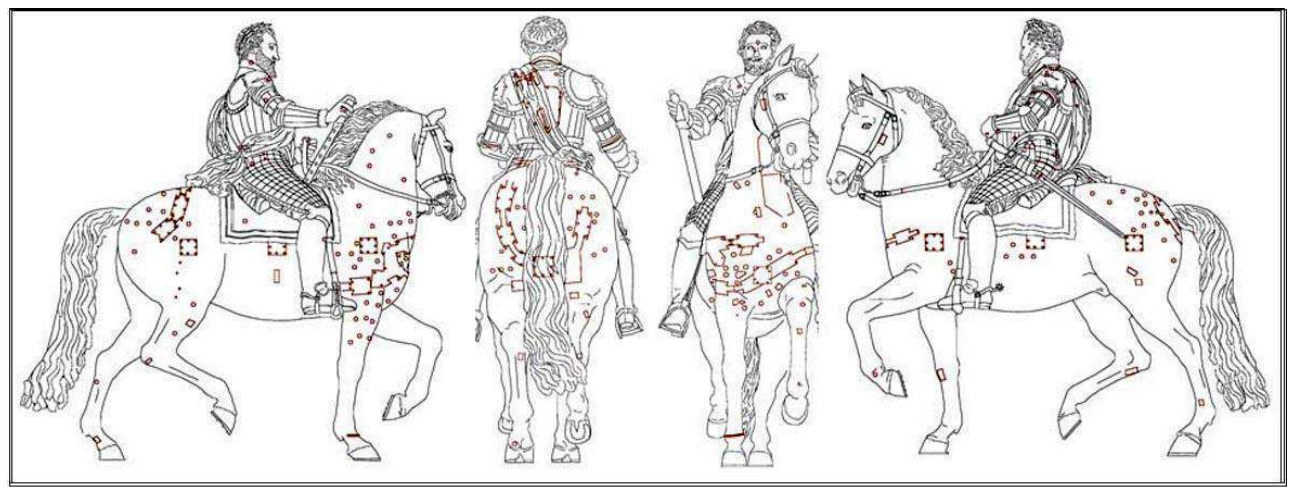

Réparations originelles de la surface

Phot. C. Usaï, 2004

La surface du cheval est marquée par de multiples pastilles qui correspondent au rebouchage des extrémités des jets et évents nécessaires au coulage du bronze. Huit patchs carrés deux à deux symétriques sur le pourtour du cheval correspondent aux reprises des orifices laissés par les barres métalliques de soutien de cette pièce monumentale de la sculpture lors de la fabrication du moule (fig. $\mathbf{n}^{\circ} \mathbf{1 5}$ ).

De nombreux empiècements assemblés ou non en queue d'aronde sont visibles sur le cheval et résultent des difficultés survenues lors du refroidissement trop rapide de la pièce monumentale qui occasionna des craquelures et nécessita des colmatages en surface. D'autres facteurs sont aussi à l'origine de ces défauts : épaisseur réduite du métal, évacuation de l'air peu efficace, cassure du moule... 
Figure 16

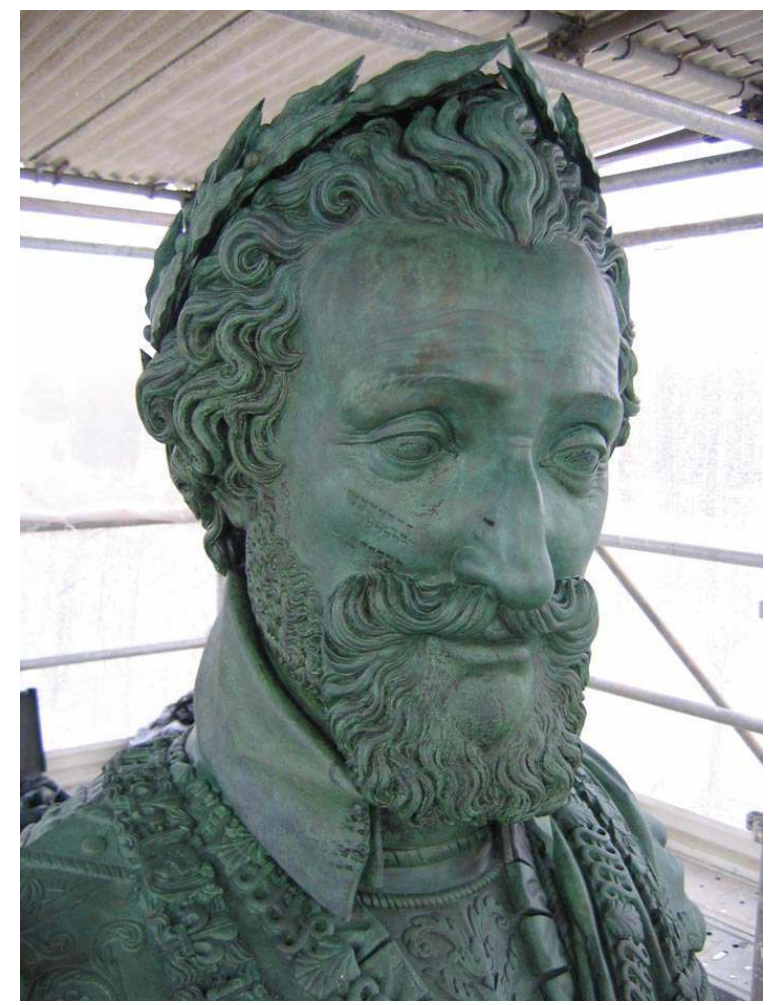

Détail de la tête après traitement et marques de vandalisme par gradine Phot. C. Usaï, 2004

La statue a été le témoin d'événements historiques et en a gardé des séquelles. Des actions de vandalisme ont laissé leurs traces. Ont été retrouvées des empreintes de grand marteau à grain d'orges au niveau du visage (fig. $\mathbf{n}^{\circ} \mathbf{1 6}$ ), du col, de la main droite du cavalier ainsi que l'amorce d'un trait de scie au niveau de l'antérieur droit du cheval. Ces empreintes témoignaient d'une volonté de destruction de la statue vraisemblablement relative aux journées révolutionnaires survenues au XIX ${ }^{\text {ème }}$ siècle. Plusieurs tirs de balles ont impacté la surface dont celui d'un projectile plus puissant ayant provoqué une écorchure profonde sur l'antérieur gauche (fig. $\left.\mathbf{n}^{\circ} \mathbf{1 7}\right)$. 


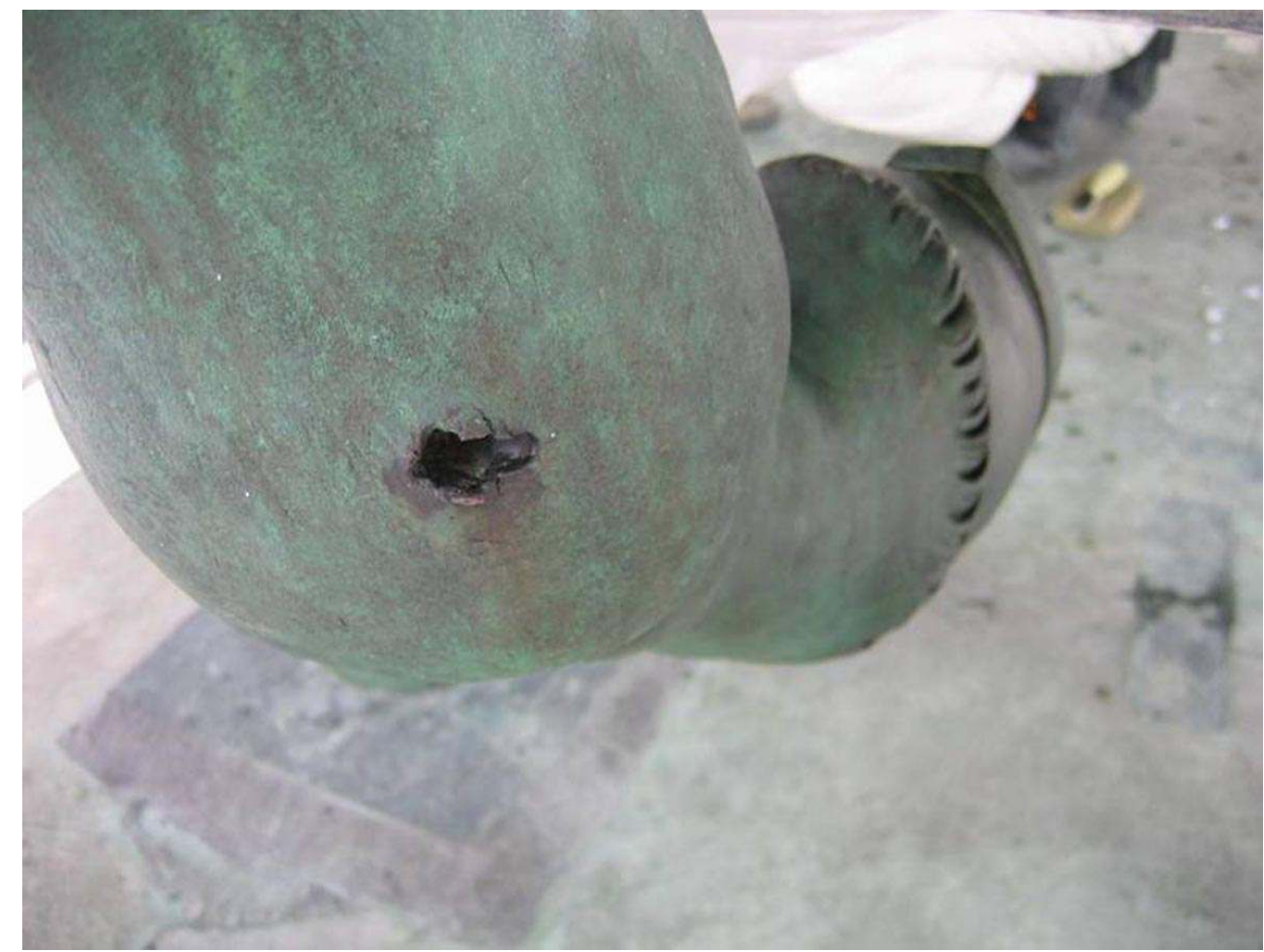

Impact de balle au niveau de l'antérieur gauche

Phot. C. Usaï, 2004

Les assemblages ont fait l'objet d'un relevé nous renseignant sur le mode d'élaboration de la statue : le cheval a été réalisé d'une seule pièce alors que le cavalier est la somme de nombreux morceaux.

37 L'observation minutieuse de la surface et le nettoyage qui l'a précédée, ont permis de révéler le travail admirable de ciselure qualifiant chaque type de surfaces avec des finitions différentes et avec une précision iconographique rare; la cuirasse de Henri IV est pour cela d'un rendu spectaculaire.

\section{Découverte des boîtes}




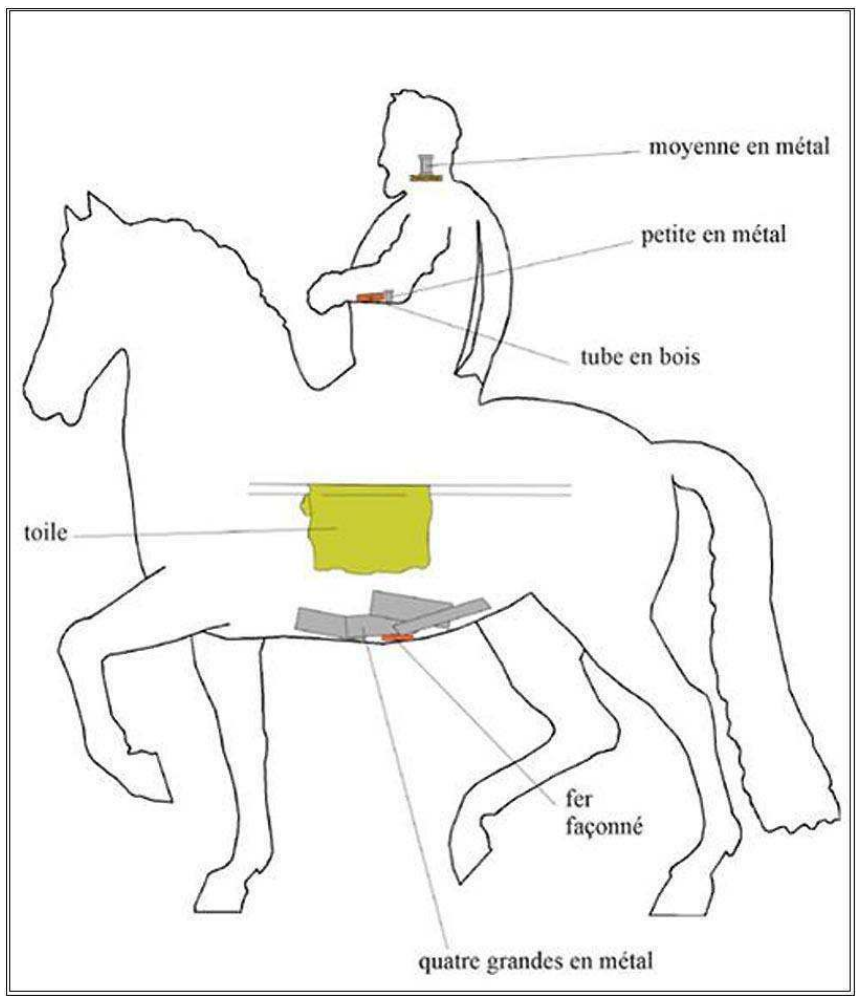

Localisation des objets à l'intérieur de la statue

Phot. C. Usaï, 2004

L'existence d'une trappe au dos du cavalier rendait possible une exploration de l'intérieur de la statue par endoscopie. Cette ouverture permit de retrouver dans l'antre de bronze, sept boîtes. Quatre boîtes rectangulaires scellées en plomb, étaient disposées dans le ventre du cheval (fig. $\left.\mathbf{n}^{\circ} \mathbf{1 8}\right)$. Le contenu de ces boîtes déposées lors de la cérémonie inaugurale et numérotées de un à quatre, était décrit dans l'ouvrage de Lafolie, conservateur des monuments publics de Paris à l'époque et qui avait été chargé de relater les étapes de l'élaboration de la statue de Henri IV. Il s'agissait de différents documents rendant hommage au Bon Roi et célébrant la restauration de la monarchie. Trois autres petites boîtes cylindriques furent découvertes dans des situations moins évidentes. Une boîte en plomb portant le nom gravé de Mesnel, le ciseleur, avait été placée en équilibre sur une planchette, dans la tête de Henri IV. Une deuxième boite similaire à la précédente mais légèrement plus petite ainsi qu'un étui en bois, ont été trouvés dans le bras gauche du cavalier. Ces trois objets inconnus pouvaient correspondre à la rumeur selon laquelle des libelles anti-royalistes avaient été introduits au sein de l'effigie de Henri IV par le ciseleur réputé Bonapartiste. 


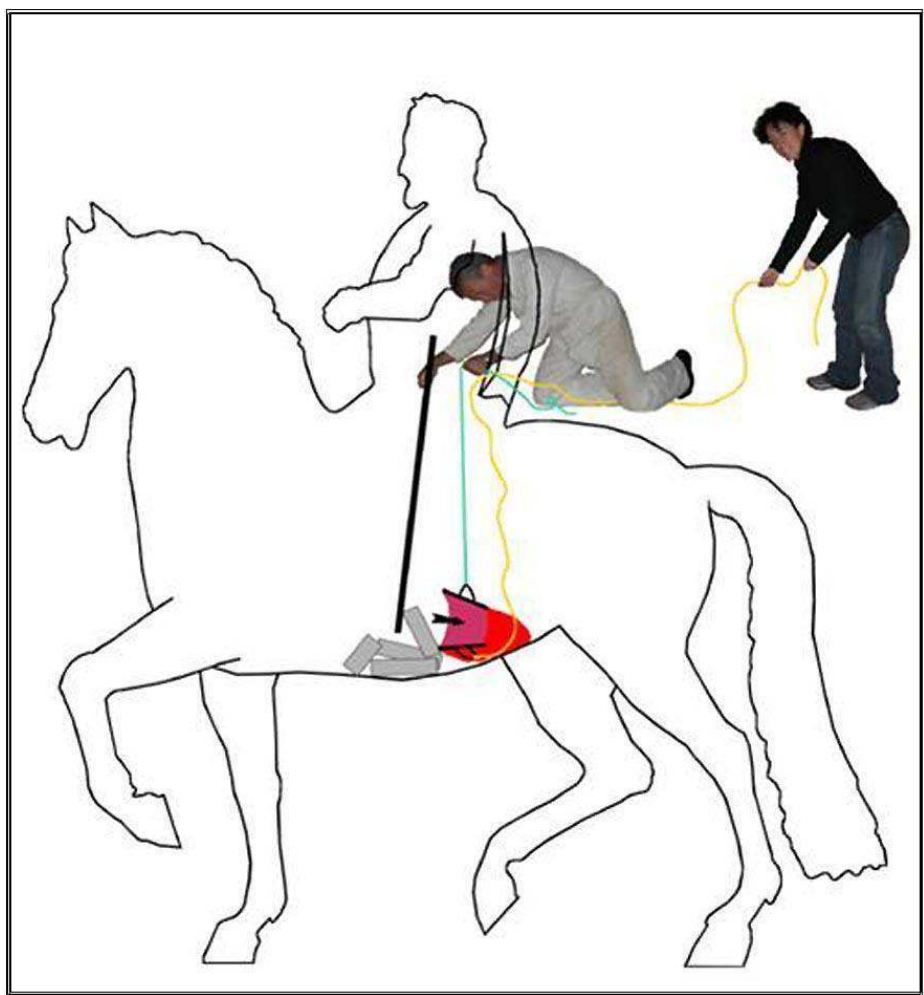

Récupération des boîtes dans le ventre du cheval

Phot. C. Usaï, 2004

La récupération des boîtes a nécessité d'imaginer d'ingénieux systèmes pour les atteindre. Les quatre boîtes rectangulaires situées au bas du ventre, ont été poussées à l'aide d'un bâton dans un sac commandé par deux cordes (fig. $\mathbf{n}^{\circ}$ 19). Deux boîtes situées dans l'avant-bras inaccessible ont été encollées à l'extrémité d'une perche pour pouvoir être retirées une fois la prise faite; l'éclairage d'une ampoule et l'image transmise par une webcam ont aidé aux manœuvres (fig. $\mathbf{n}^{\circ} \mathbf{2 0}$ ). 


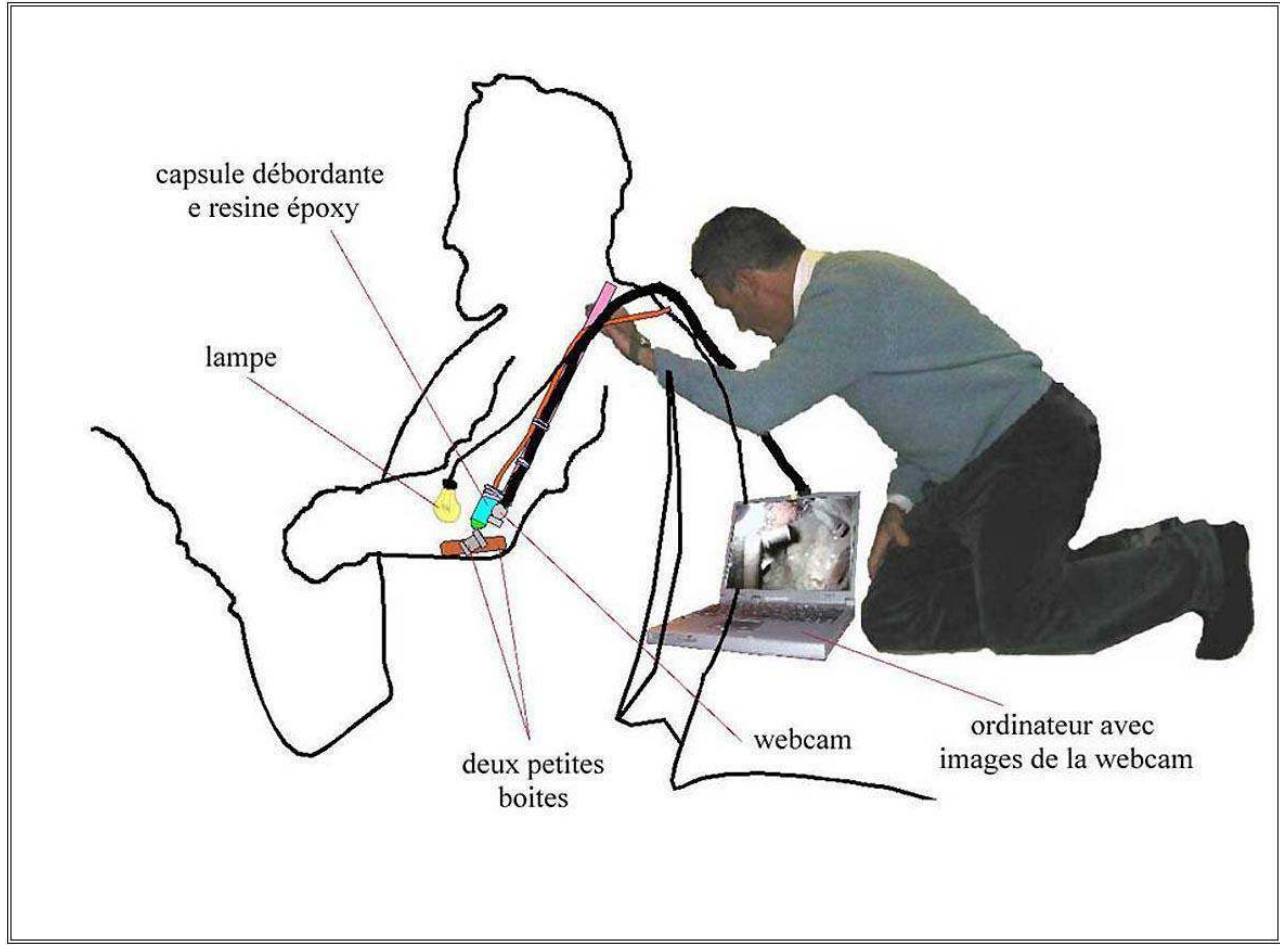

Récupération de deux boîtes dans le bras gauche Phot. C. Usaï, 2004

Devant l'intérêt de cette découverte (fig. $\mathbf{n}^{\circ} \mathbf{2 1}$ ), Renaud Donnedieu de Vabres, ministre de la Culture et de la Communication, procéda à l'ouverture officielle des boites lors d'une conférence de presse le 19 novembre 2004. 


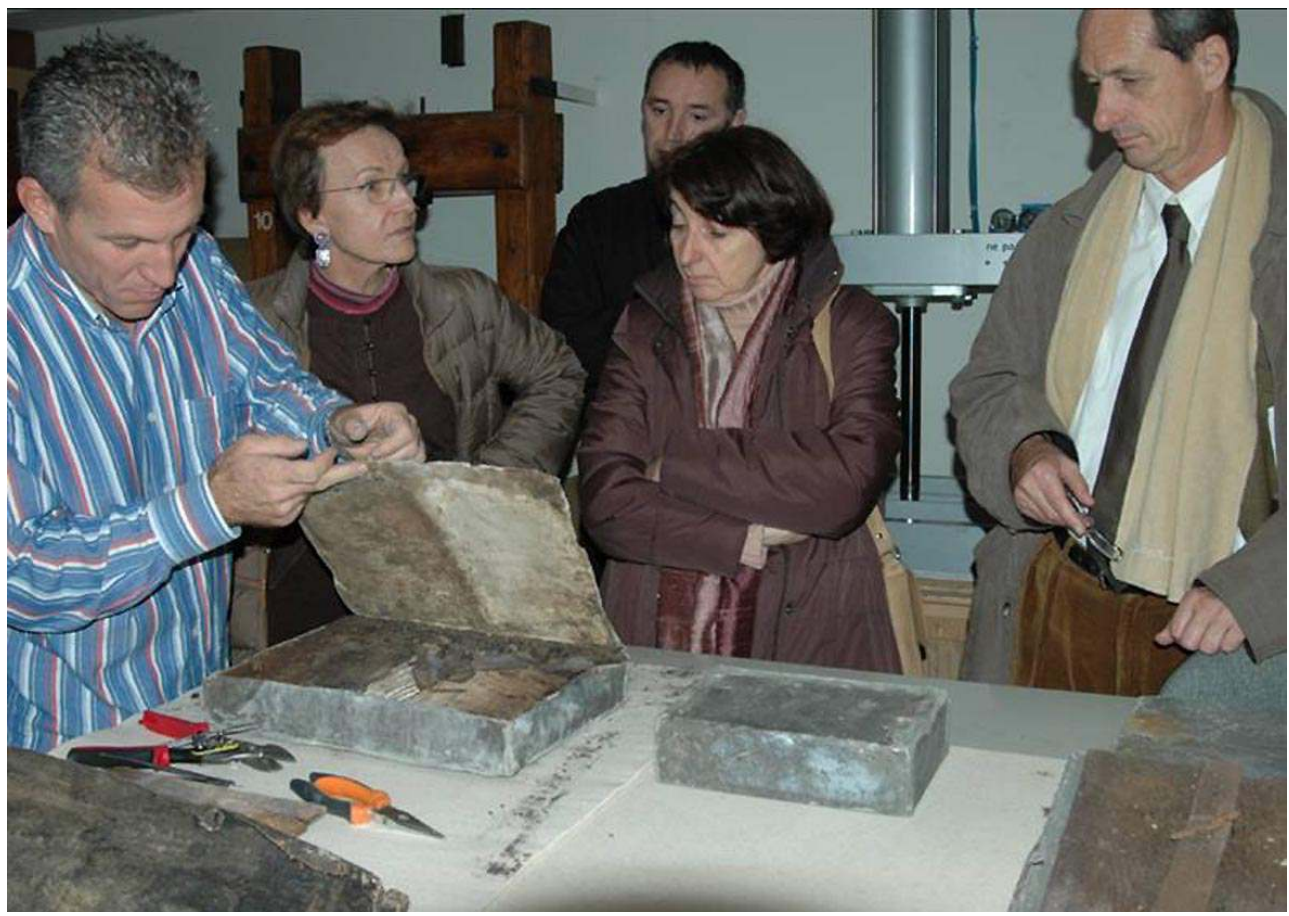

Ouverture des boîtes dans les ateliers de la bibliothèque nationale

Phot. C. Usaï, 2004

Est restée au fond du ventre, une pièce métallique d'aspect rouillé posant une énigme ; car sa forme avec une double courbure et sa dimension estimée, rappellent étrangement la garde de l'épée de la statue de Lemot (fig. $\mathbf{n}^{\circ}$ 22). La commande passée au sculpteur avait été très explicite : il s'agissait de restituer la statue de Henri IV en bronze détruite en 1792 et la nouvelle représentation devait être la plus fidèle possible au modèle. On avait une connaissance de l'œuvre originelle de Jean de Bologne à travers des représentations gravées et à travers quelques vestiges retrouvés inopinément à l'Hôtel de Ville de Paris au début du XIXème siècle. Lemot s'est aidé de ces deux sources d'informations. Ces vestiges qui comprenaient le bras gauche et la botte droite aujourd'hui conservés au Musée Carnavalet, pouvaient-ils compter d'autres pièces égarées depuis, dont la garde de l'épée d'origine? Cette pièce à valeur symbolique de relique, aurait-elle pu être glissée dans la statue de Lemot ? Le matériau ferrugineux dont est forgé ce morceau rouillé, ne contredit en rien l'appartenance à la première statue car il était courrant dans le milieu de la Renaissance en Italie que des statues de bronze soient ornées d'attributs réalisés dans un autre métal et avec une finition différente. Ces questions sans réponse, méritent cependant d'être évoquées et laissent la porte ouverte à l'hypothèse de la présence dans la statue de Lemot d'un vestige de la statue de Jean de Bologne. 
Figure 22
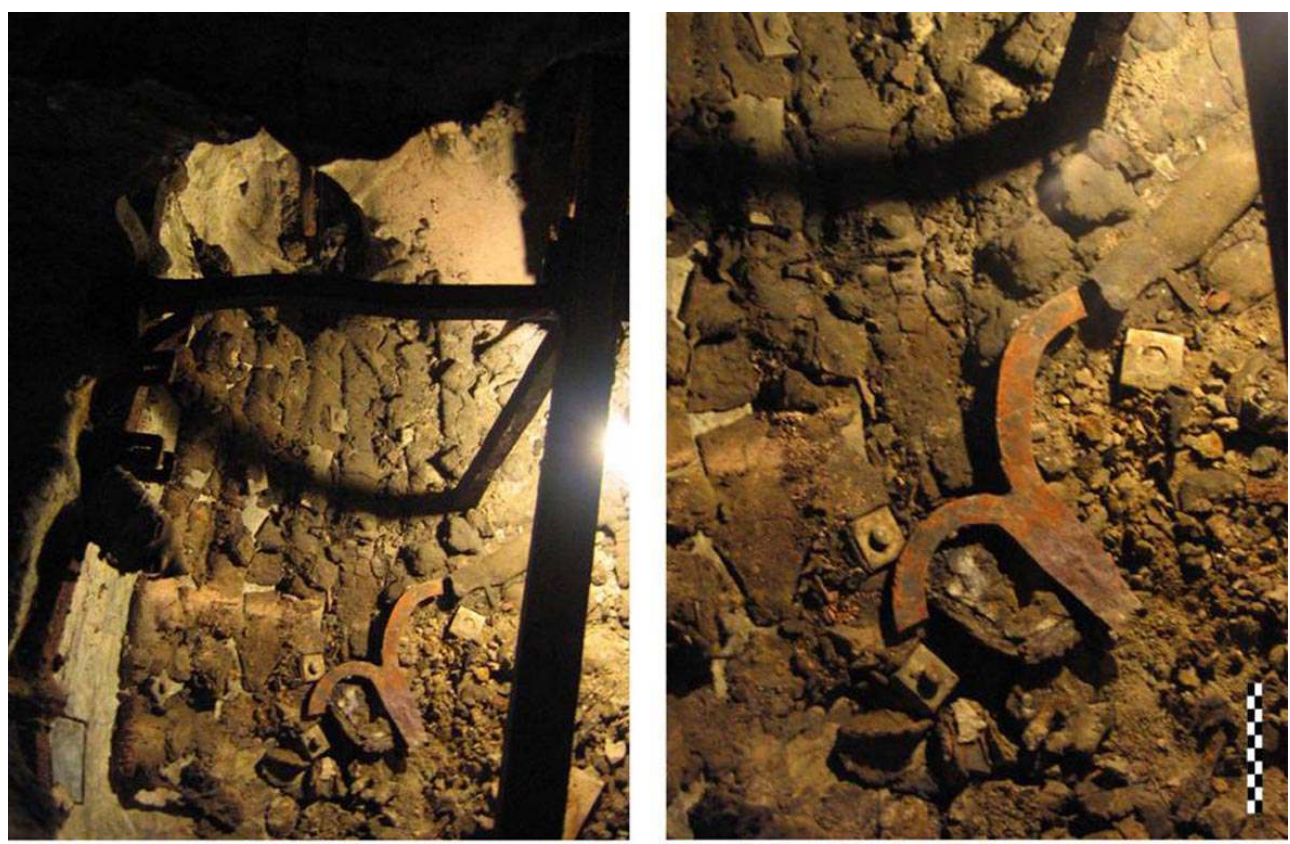

Pièce restante dont la forme rappelle la garde de l'épée de la statue

Phot. C. Usaï, 2004

\section{Observation de l'intérieur par endoscopie}

42 L'examen exhaustif par endoscopie de l'intérieur, a permis de confirmer les différents modes d'élaboration de la statue ainsi que les difficultés de mise en œuvre rencontrées. La nature des terres de fusion résiduelles, atteste deux types de façonnage selon les parties. Une terre rouge due à la présence de terre cuite pilée relevée sur les parois du cheval, prouve que l'animal a été réalisé avec la technique de la fonte à la cire perdue. Par contre, sur les parois intérieures du buste du cavalier, on trouve une terre noire riche en sable, caractéristique de la fonte au sable, méthode plus ordinaire. L'existence de nombreuses plaques boulonnées et d'une importante armature de barres métalliques dans le ventre du cheval, témoignent des besoins d'une part de remédier aux fissurations, défauts survenus lors du retrait contrarié du métal par un noyau trop dur, et d'autre part, de renforcer la cohérence du matériau de deux centimètres d'épaisseur par une structure interne forte soutenant la sous-face du ventre et rigidifiant les côtés.

\section{Restauration du socle en marbre}

L'opération s'est achevée par la restauration des emmarchements formant socle et du piédestal de la statue. Le marbre blanc dont sont composés ces éléments architectoniques, subit une érosion naturelle de ses surfaces. Ses surfaces altérées, qui ont perdu leur lustrage originelle, deviennent plus perméables aux sels et aux sulfates de cuivre contenus dans les eaux de ruissellement; ce qui entraîne des coulures vertes posant un problème esthétique. 

(Biotin-CTS à $3 \%$ ) afin d'éliminer les mousses et les micro-organismes. Puis le nettoyage a consisté en l'application de compresses d'argile (Septiolite) imbibées de carbonate d'ammonium en solution saturée. Cette opération a permis de dissoudre les sels et d'atténuer les traces vertes. Pour insister sur les parties fortement tâchées, ont été à nouveau appliquées des compresses imprégnées d'EDTA bi-sodique en solution (10\%). Mais les sulfates de cuivre ont été absorbés en profondeur et il n'a pas été possible de les faire entièrement disparaître. Quelques retouches ponctuelles par patine ont encore optimisé le résultat.

L'ensemble des joints a été repris avec un mortier composé de chaux et de poudres de marbres colorés. Le ragréage des micro-fissures a nécessité l'emploi d'un agrégat plus fin. Cette phase finale garantit la bonne étanchéité des emmarchements et du piédestal; elle permet également une meilleure harmonisation des surfaces de ces éléments architectoniques qui contribuent à la mise en valeur de la statue de Henri IV.

À l'occasion de ces travaux, la technique de taille des emmarchements a été observée. Elle est inhabituelle et dispendieuse de matière : les marches ont été taillées deux à deux, formant un bloc massif en équerre.

\section{Conclusion}

Dans sa philosophie, cette opération de restauration met l'accent sur la valeur de la matière historique, c'est-à-dire le bronze transformé par le temps dont on accepte le vieillissement. Le protocole d'intervention a cherché à répondre à la fois aux exigences de la conservation en ralentissant au mieux la corrosion du métal et à celles de l'esthétique en redonnant un aspect plus homogène à la statue. Le protocole a aussi cherché à répondre à une exigence patrimoniale en conservant la surface originelle et sa patine naturelle au lieu de lui substituer une nouvelle patine à chaud pour faire « peau neuve ».

Cette opération prouve également que le temps du chantier est encore un temps d'investigation et qu'il s'agit d'une opportunité propice aux avancées en matière de connaissances historiques et scientifiques. La découverte des boîtes et de leur contenu a suscité un nouvel intérêt autour de la statue de Lemot et de la page d'histoire qu'elle illustre. Et après tous ces siècles passés érigé en cette triomphale place, le Vert Galant peut encore se flatter de nous avoir étonnés.

\section{BIBLIOGRAPHIE}

Blengino, Jean-Michel. Inhibiteur de corrosion des bronzes, In Conservation-Restauration des Biens Culturels, ARAAFU, déc. 1990.

Dumas, Alexandre. Vingt ans après, vol. I, 1845. 
Marabelli, M., Napolitano, G. Nuovi sistemi protettivi applicabili su opere o manufatti in bronzo esposti all'aperto, In Materiali e Structure, 1991.

Leoni, M. Elementi di metallurgia applicata al restauro delle opere d'arte. Firenze : Opus Libri, 1984.

Marabelli, M., Napolitano, G. Nuovi sistemi protettivi applicabili su opere o manufatti in bronzo esposti all'aperto, In Materiali e Strutture, 1991, I, 2, p. 51-58.

Plenderleith, H. J. La Conservation des Antiquités et des Oeuvres d'Art. Paris : Eyrolles, 1966.

AA. VV. Ancient Metals. Roma : ICCROM, 1980 (il curatore è l'ICCROM).

AA. VV. Marco Aurelio, mostra di un cantiere. Roma : Arti Grafiche Pedanesi, 1984 (curato dall'Istituto Centrale del Restauro).

Brachert, T. La patina. Fiesole : Nardini, 1990.

\section{ANNEXES}

\section{Fiche technique}

Coût des travaux (socle inclus) : 49873 euros TTC

Maîtrise d'ouvrage : DRAC Île-de-France, Caroline Piel, Conservateur en Chef des

Monuments Historiques

Maîtrise d'œuvre : SDAP de Paris

Laboratoire associé : LRMH, Annick Texier, ingénieur et responsable de la section métal Durée du chantier : trois mois

\section{RÉSUMÉS}

Cette intervention de conservation du bronze de la statue de Henri IV était la première depuis sa création en 1818 : on héritait de la surface originale de l'œuvre altérée par les vicissitudes du temps et les événements historiques traversés. Le protocole de conservation se voulait respectueux de cette authenticité de la patine et a cherché à en laisser les marques tout en apportant une réponse pour contrer la corrosion active de l'alliage. C'est en conformité aux principes de conservation établis par Cesare Brandi, qu'ont été définis le nettoyage sélectif des surfaces et l'application de couches protectives sur le bronze. Par ailleurs, le moment du chantier est un moment privilégié pour observer un monument et mieux connaître son histoire particulière. Ce chantier nous a permis de redécouvrir deux ensembles de boîtes déposées dans l'antre du cheval dans des situations parfois surprenantes.

This conservation treatment for the bronze statue of Henri IV is the first since its creation in 1818. While the original surface of the work remains, it has been altered by natural and human forces; including the weathering of the material from exposure to the elements and damage caused by historic events. The conservation protocol is respectful of this authenticity of the surface patina and the marks of damage due to historical events, while developing a treatment plan to counter the active corrosion of the alloy. In keeping with the principles of conservation established by Cesare Brandi, the treatment program defines the selective cleaning of the surface and the application of several layers of a protective coating on the bronze. In addition, the 
conservation project is an opportunity to carefully study a monument to better understand its unique history. This project allowed us to rediscover two sets of boxes placed inside the cavity of the horse; an unxpected discovery.

\section{INDEX}

Mots-clés : extrémités des jets et évents, marques de vandalisme, découverte des boîtes illicites, redécouverte des boîtes officielles, compresses imprégnées d'EDTA bi-sodique, compresses d'argile imbibées de carbonate d'ammonium, Cesare Brandi, observation par endoscopie, observation de surface de la statue, protocole d'intervention sur le bronze, enjeux de la restauration du bronze, cire microcristalline, inhibiteur de corrosion, nettoyage sélectif manuel, oxyde de cuivre, sulfate de cuivre, analyse de la surface du bronze, patine naturelle du bronze, altération du bronze dans un environnement urbain

\section{AUTEURS}

\section{STÉPHANIE CELLE}

Architecte-urbaniste de l'État, conservateur du monument (2003-2009), actuellement conservateur des monuments nationaux. stephanie.celle@monuments-nationaux.fr

\section{CARLO USAI}

Restaurateur, enseignant à l'Istituto Centrale per il Restauro, Rome. carlousai@iol.it 